\title{
Photoluminescence Characterization of Cadmium Sulphide (CdS) Nanowires for Polarization Studies
}

\author{
Shripriya Poduri ${ }^{1}$, Mitra Dutta ${ }^{1,2} \&$ Michael Stroscio ${ }^{1,2,3}$ \\ ${ }^{1}$ Department of Electrical and Computer Engineering, University of Illinois at Chicago, Chicago, IL 60607, \\ USA \\ ${ }^{2}$ Department of Physics, University of Illinois at Chicago, Chicago, IL 60607, USA. \\ ${ }^{3}$ Department of Bioengineering, University of Illinois at Chicago, Chicago, IL 60607, USA \\ Correspondence: S. Poduri, Department of Electrical and Computer Engineering, University of Illinois at Chicago, \\ Chicago, IL 60607, USA. E-mail: shripriyapoduri@gmail.com
}

Received: September 30, 2017

Accepted: October 20, 2017 Online Published: November 10, 2017

doi:10.5539/apr.v9n6p26

URL: https://doi.org/10.5539/apr.v9n6p26

\begin{abstract}
In this paper, the polarizing properties for CdS nanowire arrays were explored for their potential use in the design of nanowire based polarizers and optical switches. These free standing cadmium sulphide (CdS) nanowires were grown in anodized aluminum oxide (AAO) template via de electrodeposition. Raman and photoluminescence (PL) measurements were investigated for parallel and perpendicular polarization with two orientations of the sample having light propagating parallel to the nanowire axis in one orientation and light propagating perpendicular to the nanowire axis in other orientation. Polarization-sensitive measurements show strong polarization anisotropy in the photoluminescence (PL) intensity measurements observed in parallel and perpendicular orientation to the long axis of a nanowire. The measured PL ratio, $\rho$, for parallel to perpendicular orientation was around $0.80-0.85$ which shows strong polarization anisotropy for the grown CdS nanowires. Strong peaks of A1 (TO) at $235 \mathrm{~cm}^{-1}$, E2 mode at $255 \mathrm{~cm}^{-1}$ along with $1 \mathrm{LO}$ (longitudinal optical) at $303 \mathrm{~cm}^{-1}, 2$ LO peak at $604 \mathrm{~cm}^{-1}$ of the CdS nanowires were seen with different polarizations for Raman spectral studies. These polarization studies show that these dc electrodeposited grown CdS nanowire arrays are well suited for uses in polarization-based nanoscale devices such as in optical switches, and high performance photodetectors.
\end{abstract}

\section{Introduction}

With the advent and rapid development of nanotechnology, semiconductor nanowires (NWs) have become essential components for future "nano-devices". Semiconductor nanoscale structures have generated a great deal of interest owing to their strong confining potentials for electrons and holes and the associated quantum effects (Dutta et al., 2014; Morales \& Lieber, 1998; Seminario, 2014; K. Xu et al., 2014). Therefore, semiconductor nanowire devices are being widely used as photo-detectors (Gu et al., 2005; Qian, Gradecak, Li, Wen, \& Lieber, 2005), sensors (Brenneman, Poduri, Stroscio, \& Dutta, 2013; S. D. Poduri, 2010; Souvik Mukherjee, 2015), lasers (Agarwal, Barrelet, \& Lieber, 2005; Duan, Huang, Agarwal, \& Lieber, 2003), transistors (Farid et al., 2015; Xiang et al., 2006), optical switches (Kind, Yan, Messer, Law, \& Yang, 2002), etc. Recent research in the field of semiconductor nanowires have revealed a lot of interesting properties such as polarization sensitivity (Z. Y. Fan et al., 2004; Shan, Liu, \& Hark, 2006), size-dependent photoluminescence (S. Poduri \& Stroscio, 2015; Samuelson et al., 2004), quantum dots in nanowires for LEDs (light emitting diodes) (Gudiksen, Wang, \& Lieber, 2002), etc. These interesting properties of semiconductor nanowire devices are being employed in both scientific and industrial applications. One of the most important II-IV semiconductor compounds, cadmium sulphide (CdS) was selected because it is used widely in optoelectronic devices due to its direct band gap of 2.42 $\mathrm{eV}$ and its excellent semiconducting properties.

Cadmium sulphide nanowires have significant advantages such as high aspect ratio, huge surface to volume ratio leading to higher sensitivity of excitons to surface states and defects as compared to their bulk counterpart, and excellent electron emission or absorption property in optoelectronic applications(Mazouchi, Poduri, \& Dutta, 2014; Poduri, Dutta, \& Stroscio, 2014). But the current understanding of using grown CdS nanowires as polarizers is limited and therefore the polarizing properties of grown CdS nanowires have been explored as reported in this paper to demonstrate their potential uses in polarization-based nanoscale devices. DC 
electrodeposition of CdS nanowires is not very commonly done for samples with very narrow pore diameters. However, in this paper CdS nanowires were grown by using a simple dc electrodeposition process into the narrow pores of 10-60 nm diameters by overcoming the challenges faced during the growth of CdS nanowires. The challenge faced during the growth of electrodeposited CdS nanowires is barrier layer removal which leads to poor crystalline structure of nanowires. The growth of decent quality CdS nanowires was achieved, even though it has been stated in (Routkevitch, Bigioni, Moskovits, \& Xu, 1996) that DC electrodeposition was not an easy process for growing nanowires in narrow pores. The method of barrier layer removal is also discussed in this paper. Polarization studies with DC electrodeposited grown CdS nanowires has not been performed so far.

The polarization anisotropy feature employed in high performance nanowire polarizers can be explained in terms of the large dielectric difference between these freestanding nanowires and the surrounding environment. This property of polarization anisotropy is employed here to demonstrate its potential use in the application of high performance polarization-based sensitive nanoscale based optical switches, and polarizers (Wang, Gudiksen, Duan, Cui, \& Lieber, 2001).

\section{Experimental Details}

\subsection{Growth of Anodized Alumina Templates and Nanowires}

Recently, a lot of research has been done in growing free standing nanowires on a substrate in a controlled fashion (Anyebe et al., 2015; Taşaltın, Öztürk, K1lınç, Yüzer, \& Öztürk, 2010; Zhou et al., 2009). Growing free standing nanowires has always been a challenge but the easiest and most economical way to grow free standing nanowires is through self assembly of electrodeposited CdS nanowires in an anodized alumina template. CdS nanowires were grown in anodized aluminum sheet metal of thickness of $500 \mu \mathrm{m}$. The anodized template was prepared using the protocol given in Routkvitch et al. (1996). The aluminum (Al) metal sheet (99.9 \% purity) of $2 " \times 0.5 " \times 0.020$ " dimensions was cleaned by ultrasonication in acetone for 1 hour followed by ultrasonication in methanol for 1 hour. These $\mathrm{Al}$ metal sheets were then electropolished in perchloric acid and ethanol solution having volume ratio of $1: 5$ at $0{ }^{\circ} \mathrm{C}$ for 15 minutes. After electro-polishing, the templates were anodized at a potential of $40 \mathrm{~V}$ for a minimum of 16 hours and the temperature was maintained at $10{ }^{\circ} \mathrm{C}$ throughout the process. After the first anodization step, the oxide layer was removed by immersing the sample in a solution of $1.8 \% \mathrm{wt}$ chromic acid and $6 \% \mathrm{wt}$ phosphoric acid at $60{ }^{\circ} \mathrm{C}$ for $4-5$ hours. The second anodization was performed with the same conditions as that of the first anodization step but the time of the anodization this time was only 20 minutes. The barrier layer was removed by dipping the anodized template in $0.5 \mathrm{M}$ phosphoric acid for 75 minutes at room temperature. The electrolyte for CdS electrodeposition was a solution of dimethyl sulfoxide (DMSO) comprising of $0.055 \mathrm{M} \mathrm{CdCl} 2$ and $0.19 \mathrm{M}$ elemental sulfur (Xu et al., 2000; Yang et al., 2002). The temperature of the electrolyte was $120-150{ }^{\circ} \mathrm{C}$ during the electrodeposition growth and current density of $15 \mathrm{~mA} / \mathrm{cm} 2$ was applied for 7-10 s. The deposition rate was $25-30 \mathrm{~nm} / \mathrm{s}$. After the DC electrodeposition, the sample was rinsed in hot DMSO followed by acetone and cleaned in deionized water. The AAO template was dissolved in $1 \mathrm{M} \mathrm{NaOH}$ solution at room temperature for 8 min to liberate the $\mathrm{CdS}$ nanowires.

\subsection{Photoluminescence and Raman Experimental Set Up}

To study the polarization anisotropy, PL studies were performed with the continuous wave He-Cd laser of wavelength $441 \mathrm{~nm}$ for parallel and perpendicular polarization with two orientations of the sample having light propagating parallel to the nanowire axis in one orientation as shown in Figure 1 and light propagating perpendicular to the nanowire axis in other orientation as shown in Figure 2. The PL and Raman intensities were recorded at room temperature using He-Cd laser of wavelength $441 \mathrm{~nm}$ and Acton 2500i spectrometer. The polarization filter used was LPVISE100 purchased from Thor labs Inc. For measuring Raman spectra, holographic notch filters were used to do the Raman measurements, and the Raman system was calibrated by measuring the phonon peak on silicon first. PL and Raman intensities were recorded five times for parallel and perpendicular polarization with two orientations of the sample having light propagating parallel to the nanowire axis in one orientation and light propagating perpendicular to the nanowire axis in other orientation.

The phononic modes of these polarizations were also studied to investigate further the anisotropy present in the CdS nanowires. Therefore, Raman characteristics were recorded for two orientations of the sample with light propagating parallel $(\mathrm{I})$ to the nanowire axis in one orientation as shown in Figure 1 and light propagating perpendicular $(\perp)$ to the nanowire axis in other orientation as shown in Figure 2. Raman intensities were recorded for both parallel $\mathrm{Z}(\mathrm{XX}) \mathrm{Z}$ and perpendicular polarization $\mathrm{Z}(\mathrm{XY}) \mathrm{Z}$ of the laser. The notations $\mathrm{Z}(\mathrm{XY}) \mathrm{Z}$ described in this paper are to designate the directions. The directions inside the parentheses denote the polarizations of the incident and scattered beams, respectively. The directions preceding and following outside 
the parentheses indicate the respective directions of the incident beam and scattered beams (Arguello, Rousseau, \& Porto). In addition, the electron phonon coupling interactions were studied by performing Raman spectroscopy at different angles of laser polarization with the same Raman experiment set up.
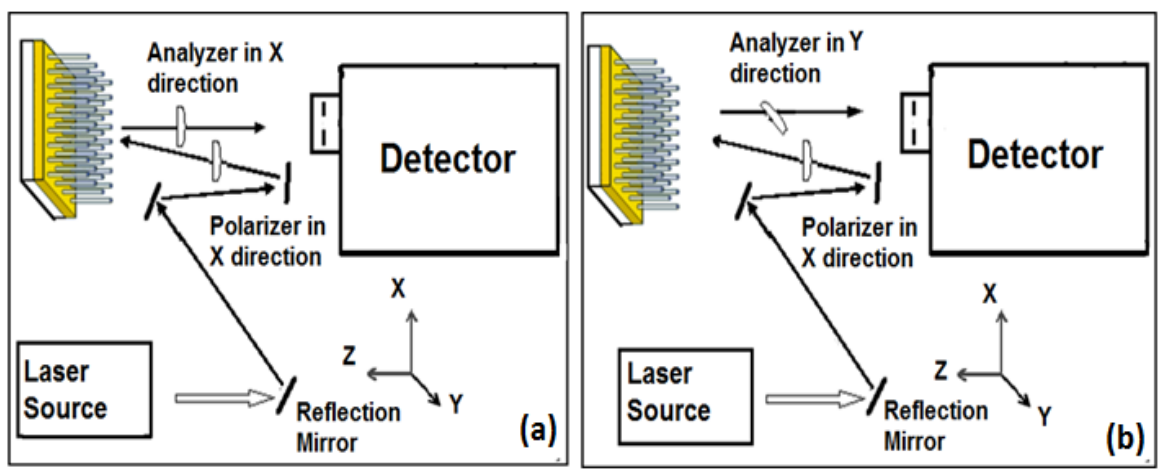

Figure 1. (a) Parallel and (b) perpendicular polarization with light propagating parallel to the nanowire axis
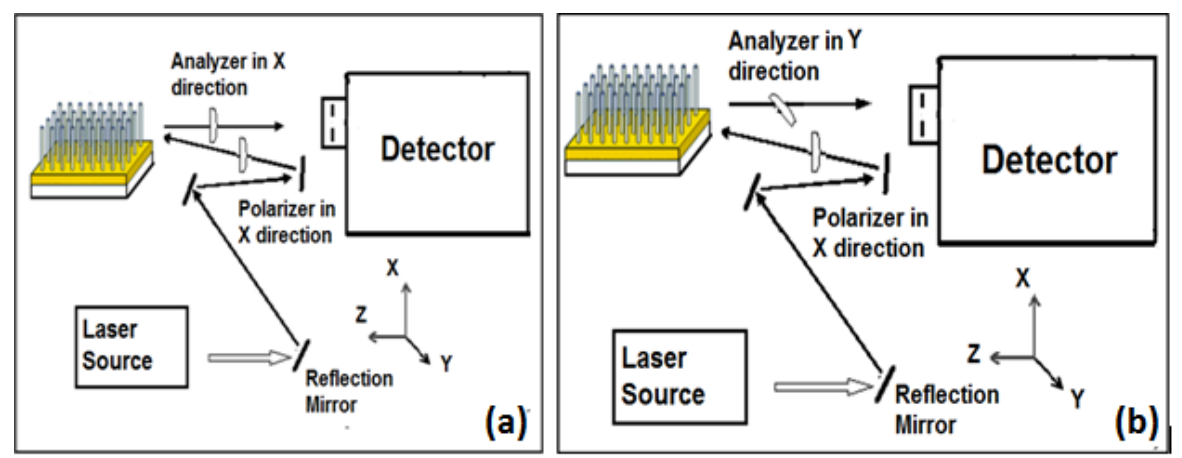

Figure 2. (a) Parallel and (b) perpendicular polarization with light propagating perpendicular to the nanowire axis

\section{Results and Discussions}

\subsection{PL Measurements Results}

The polarization anisotropy in PL measurements between the free standing nanowires and their surrounding environment can be explained by a model where the nanowire can be assumed as an infinitely long dielectric cylinder in vacuum or air, since the wavelength of the exciting light is much greater than the wire diameter. When the incident field is polarized parallel to the cylinder, the electric field inside the cylinder is not attenuated. But, when polarized perpendicular to the cylinder, the electric field amplitude is attenuated (Ruda \& Shik, 2005) according to (1)

$$
E_{i}=\frac{2 \varepsilon_{0}}{\varepsilon+\varepsilon_{0}} E_{e}
$$

where Ei is the electric field inside the cylinder, Ee the excitation field, and $\varepsilon$ is the dielectric constant of the cylinder (which is nanowire in our case) and $\varepsilon_{0}$ is the dielectric constant of the surrounding (air). For $\varepsilon>\varepsilon_{0}$, the incident light in the direction of polarization parallel to the nanowire axis creates an electric field of high frequency in the nanowire. The PL spectra can be recorded from a number of individual wires as a function of excitation or emission polarization (Ils et al., 1995; Vouilloz et al., 1998). On average, the excitation and emission polarization ratio is given by (2): 


$$
\rho=\frac{I_{\|}-I_{\perp}}{I_{\|}+I_{\perp}}
$$

The intensities parallel $\left(\mathrm{I}_{||}\right)$and perpendicular $\left(\mathrm{I}_{\perp}\right)$ to the wire can be measured with the PL measurements at different angles of orientation. The anisotropy in the photoluminescence when a nanowire array is excited with a beam polarized parallel and perpendicular to its long axis has been explained theoretically by Ruda and Shik (2005). They considered an array of dipoles randomly oriented in a dielectric cylinder and calculated the Poynting vector outside the cylinder resulting from the incoherent dipole emission. Ruda and Shik found that under this assumption the anisotropy contrast of the emission is given by

$$
\frac{\mathrm{I}_{\|}}{\mathrm{I}_{\perp}}=\frac{\left(\varepsilon+\varepsilon_{0}\right)^{2}+2 \varepsilon_{0}^{2}}{6 \varepsilon_{0}^{2}}
$$

The large polarization response is due primarily to the large dielectric difference between the nanowire and its air or vacuum surroundings. Calculating the polarization anisotropy, $\rho$, using this equation for the CdS nanowires gives 0.87 (0.89) using dielectric constant $\varepsilon_{\perp}\left(\varepsilon_{||}\right)$of CdS as 8.5 (9.25) from Table 1 (Lee, Kim, Dutta, \& Stroscio, 1997). Therefore, to study the polarization anisotropy, PL studies were done with and without the polarization filter and PL intensity were recorded for parallel and perpendicular polarization with two orientations of the sample having light propagating parallel to the nanowire axis in one orientation and light propagating perpendicular to the nanowire axis in other orientation.

Table 1. Parametric constants of CdS (Lee et al., 1997)

\begin{tabular}{ll}
\hline Parameter & Value \\
\hline Band gap $(\mathrm{eV})$ & 2.42 \\
Dielectric constant at low frequency along z $\left(\boldsymbol{\varepsilon}_{\|}\right)$ & 9.25 \\
Dielectric constant at low frequency perpendicular to z $\left(\boldsymbol{\varepsilon}_{\perp}\right)$ & 8.7 \\
Dielectric constant at high frequency along z $\left(\boldsymbol{\varepsilon}_{\infty \| \mid}\right)$ & 5.5 \\
Dielectric constant at low frequency perpendicular to z $\left(\boldsymbol{\varepsilon}_{\infty \perp}\right)$ & 5.53 \\
\hline
\end{tabular}

The CdS nanowires were grown in AAO templates and were examined under JEOL 7500 Scanning Electron Microscope (SEM) to ascertain the growth of CdS nanowire. The SEM micrograph of the CdS nanowires is shown in Figure 3.

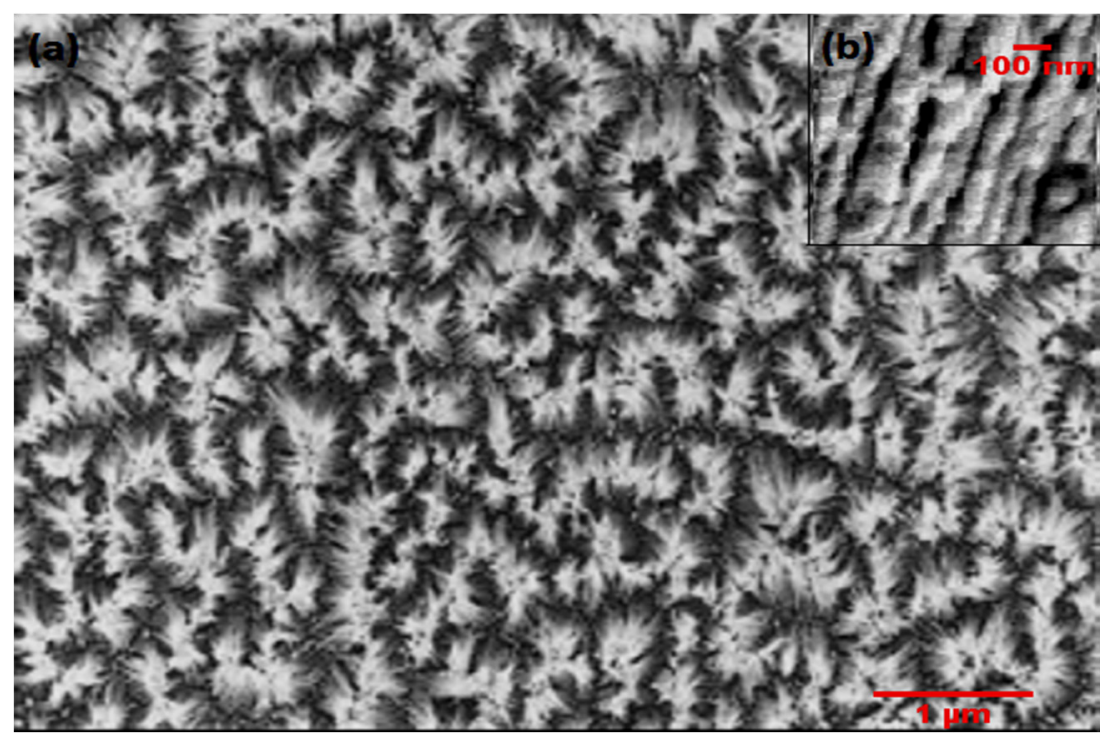

Figure 3. (a) Top view SEM of CdS nanowires grown in anodized alumina sheet of $500 \mu \mathrm{m}$. (b) SEM micrograph of tilted side view of $\mathrm{CdS}$ nanowires at a higher magnification

Raman and PL intensities were recorded for parallel and perpendicular polarization with two orientations of the sample as shown in Figure 1 and 2. The measured PL with and without the polarization filter for parallel and perpendicular polarization is shown in Figure 4. Therefore, the measured polarization ratio, $\rho$, is given by 
equation (3) by taking the intensity values from Figure 4 (a) is 0.85 when light is propagating along the length of the nanowire and 0.80 when light is propagating perpendicular to the length of the nanowire. The measured PL ratio which is 0.80 or 0.85 is in agreement with our calculated PL ratio 0.87 . Therefore, it can be said that the $\mathrm{CdS}$ nanowires would be suitable for polarized based applications. The reported phonon frequencies observed for CdS wurtzite crystal in (Arguello et al.) are given in Table II. Furthermore, to evaluate the crystal quality for structural distortions, the photoluminescence power dependence was investigated for the determination of underlying excitionic emission or recombination methods, PL was performed after annealing with different neutral density filters (purchased from Thorlabs Inc.) (Schmidt, Lischka, \& Zulehner, 1992). The PL spectral intensity for various transmission laser power density percentages after annealing has been shown in Figure 5(a) and the plot for the PL intensity with respect to various transmission laser power density percentages is in Figure 5 (b). The plot of PL intensity area under the curve with respect to different excitation power is a near linear plot with a positive slope which proves that the emission was mainly from excitons and the recombination were mostly from band to band and there were minimal structural distortions as well. Also, to study more about the structural distortions and phononic structure, Raman spectroscopy studies were performed in different angles of laser polarization. All the modes were seen in Raman spectroscopy which is in agreement with CdS nanowires structures attributing to decent quality $\mathrm{CdS}$ nanowires with minimal structural defects.
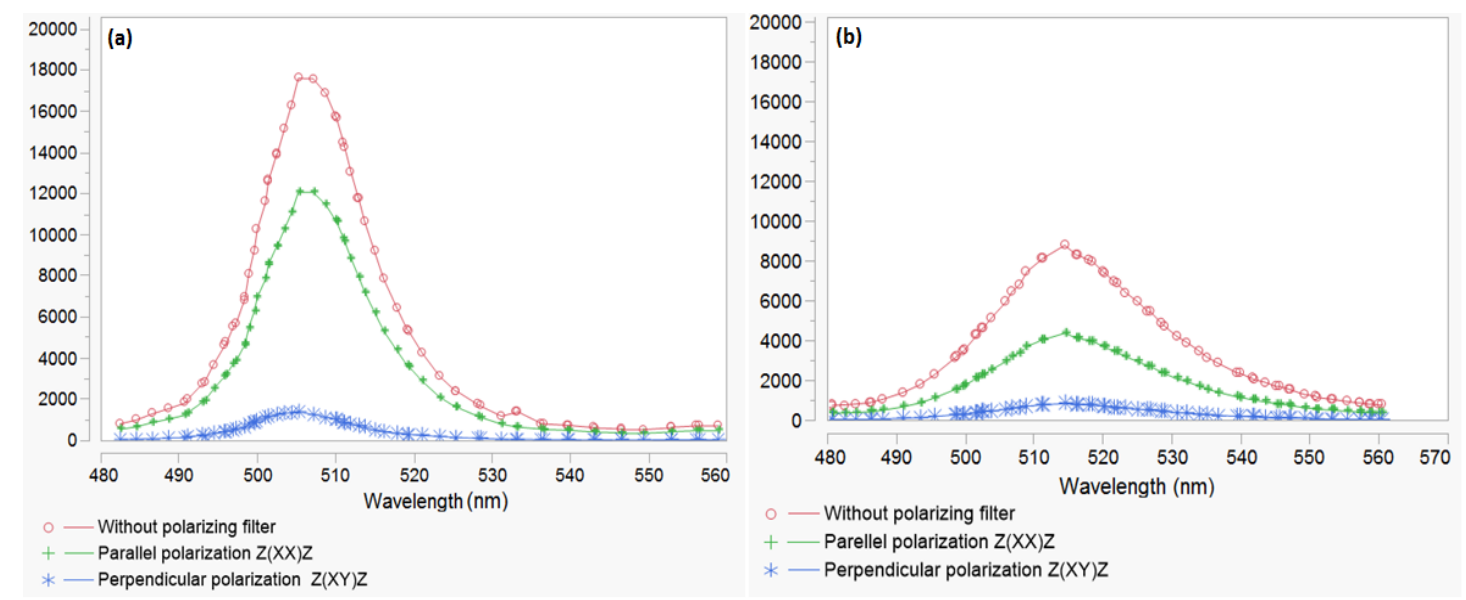

Figure 4. (a) PL peak intensity variation measured when light is propagating along the length of the nanowire along Z. (b) PL peak intensity variation measured when light is propagating along Z but perpendicular to the length of the nanowire. The measured PL ratio is 0.80

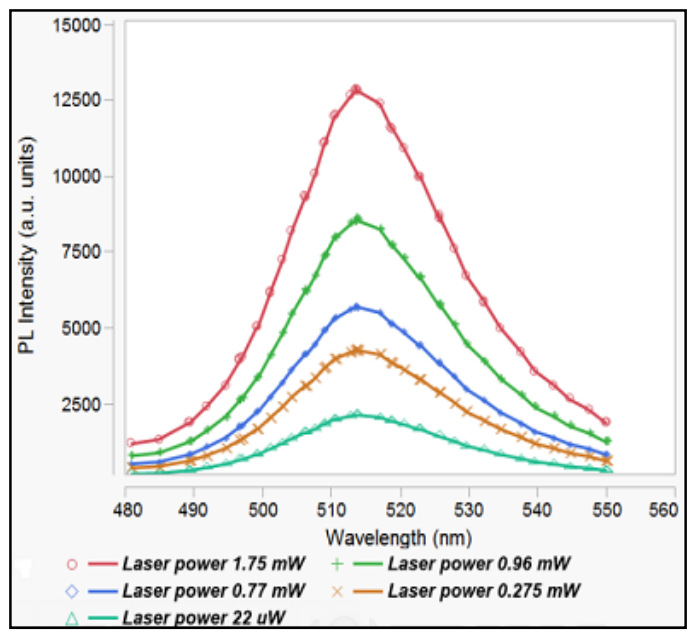

(a)

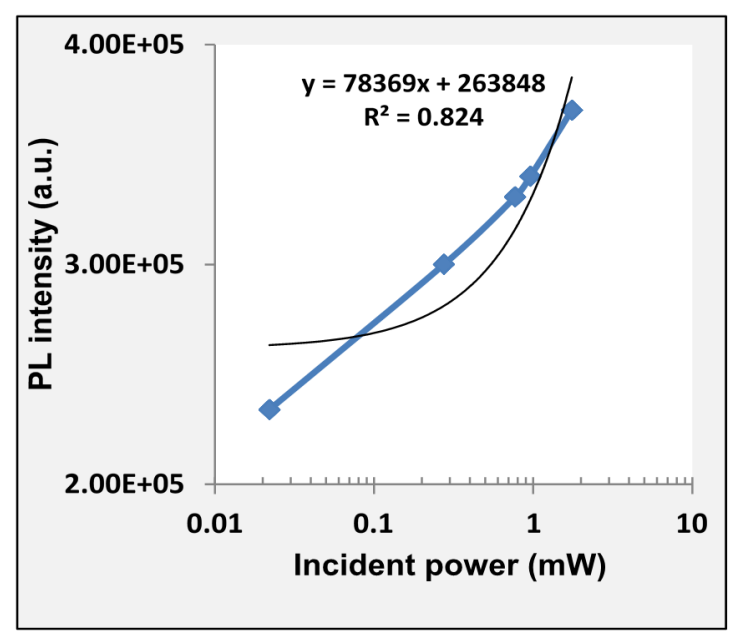

(b)

Figure 5. (a) PL spectra of CdS nanowires with different laser excitation power using neutral density filters (b) PL intensity area variation vs. different laser excitation power using neutral density filters 
Figure 5 shows the Raman spectra of CdS nanowires using the polarization filter when light is propagating along the length of the wire along Z. Peaks in intensity were observed at A1 (TO) at $235 \mathrm{~cm}-1$ for parallel polarization and at E2 at $258 \mathrm{~cm}-1$ for perpendicular polarization along with $1 \mathrm{LO}, 2 \mathrm{LO}$ at 309 and $607 \mathrm{~cm}-1$ respectively. These peaks are in accordance with the CdS values of transverse and longitudinal mode peaks (Fan et al., 2007). Also, Raman spectral studies were performed with the polarization filter when light was propagating perpendicular to the length of the wire. The peaks were found at peak at E1 (transverse) at $243 \mathrm{~cm}-1$ for parallel polarization of incident light as shown Figure 6 (a) and at quasi A1 (transverse) at $239 \mathrm{~cm}-1$ for perpendicular polarization as shown in Figure 6 (b) along with 1 LO, 2 LO at 310 and $604 \mathrm{~cm}-1$, respectively.

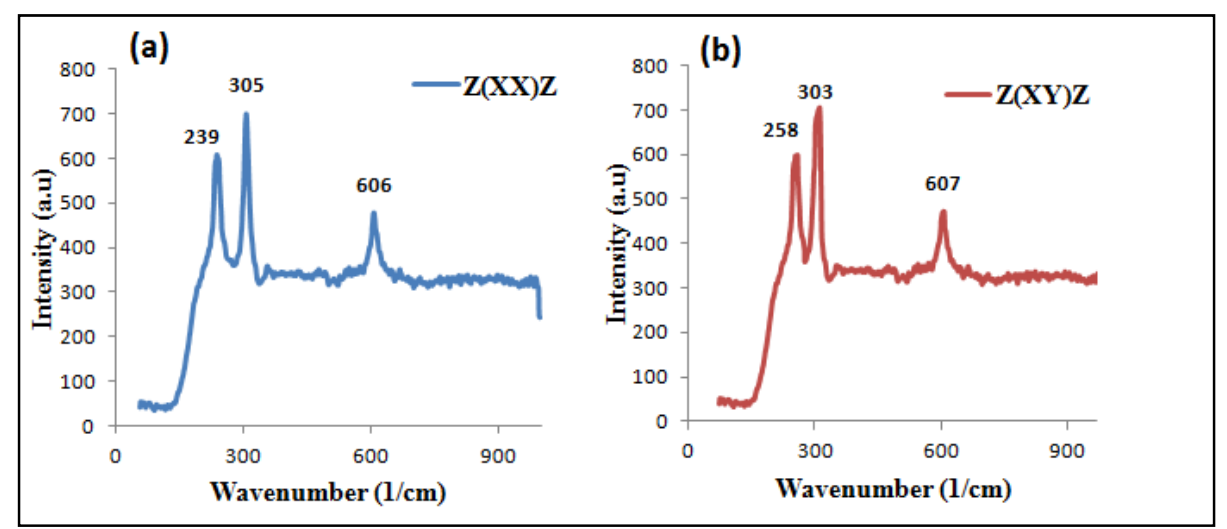

Figure 6. Raman using the polarization filter with peak at A1 (TO) at $239 \mathrm{~cm}^{-1}$ for parallel polarization in (a) and at $\mathrm{E} 2$ at $258 \mathrm{~cm}^{-1}$ for perpendicular polarization in (b) along with $1 \mathrm{LO}, 2 \mathrm{LO}$ at 305 and $607 \mathrm{~cm}^{-1}$ respectively when light is propagating along the length of the wire along $\mathrm{Z}$

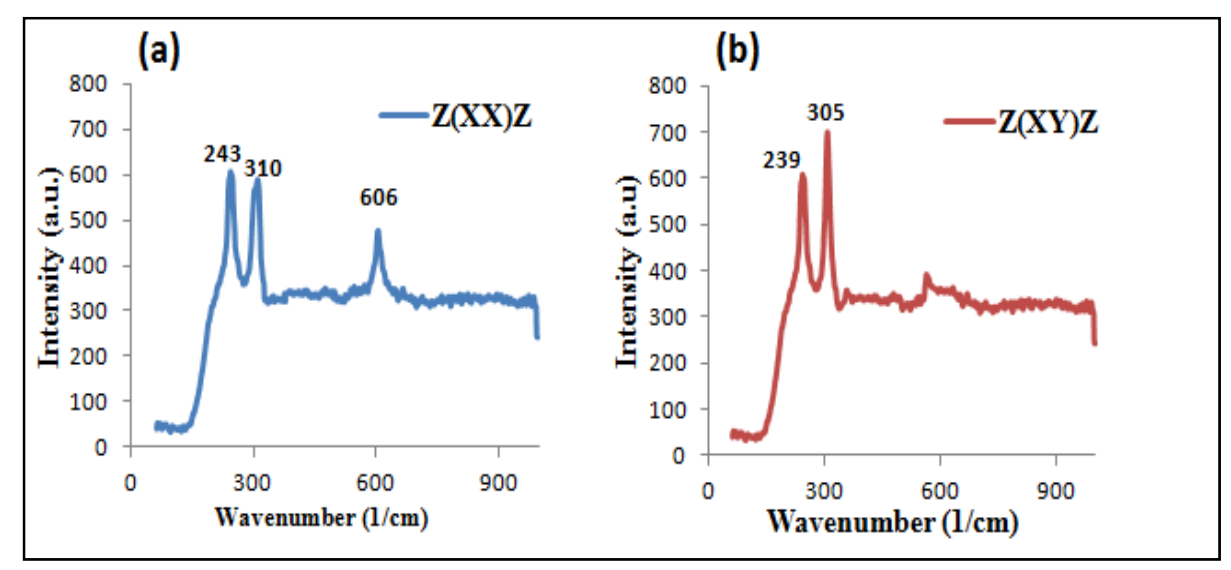

Figure 7. Raman using the polarization filter with peak at $\mathrm{E} 1$ (transverse) at $243 \mathrm{~cm}^{-1}$ for parallel polarization in (a) and at quasi A1 (transverse) at $239 \mathrm{~cm}^{-1}$ for perpendicular polarization in (b) along with $1 \mathrm{LO}, 2 \mathrm{LO}$ at 310 and $606 \mathrm{~cm}^{-1}$, respectively when light is propagating along $\mathrm{Z}$ but perpendicular to the length of the wire

The observed peaks were slightly different for CdS NWs than the reported frequencies of CdS bulk crystals given in Table 2. It was observed experimentally that the magnitude of Huang Rhys factor, which signifies the electron phonon coupling strength, decreases with the increasing angles of laser polarization. The Huang Rhys factor is the ratio of normalized Raman intensity of $2 \mathrm{LO}$ peak with respect to $1 \mathrm{LO}$ peak and is computed from the experimental data of Raman spectrum shown in Figure 6. The electron phonon coupling strength $\mathrm{S}$ is estimated by measuring the Raman intensity of the $2 \mathrm{LO}$ phonon with respect to that of the LO phonon (Fan et al., 2008) where $\mathrm{S}$ is the Huang Rhys factor. The calculation of the Huang Rhys factor for the experimental data shown in Figure 8 is given in Table 3. 
Table 2. Optical phonon modes of wurtzite CdS crystal $\left(\mathrm{cm}^{-1}\right)$ (Lee et al., 1997)

\begin{tabular}{ll}
\hline Optical mode & CdS \\
\hline E2 & 43 \\
E2 & 256 \\
A1 & 234 \\
Quasi- A1 (transverse) & 240 \\
Quasi- E1 (transverse) & 239 \\
E1 (transverse) & 243 \\
A1 (longitudinal) & 305 \\
Quasi- A1 (longitudinal) & 306 \\
Quasi- E1 (longitudinal) & 306 \\
E $_{1}$ (longitudinal) & 307 \\
\hline
\end{tabular}

Table 3. Huang Rhys factor values computed from experimental data given in Figure 6

\begin{tabular}{ll}
\hline Angle $\boldsymbol{\theta}$ in degrees & Huang Rhys parameter, $\mathbf{S}$ \\
\hline 0 & 1.1 \\
30 & 0.88 \\
60 & 0.78 \\
90 & 0.67 \\
\hline
\end{tabular}

It is observed that the Raman intensity is dependent on the angle of the laser polarization, the orientation of the nanowires and also the electron phonon interaction in the nanowires. Also, with polarization anisotropy present in the nanowires, the symmetry of the phonon modes is still in agreement with the phononic structure of $\mathrm{CdS}$ nanowires with minimal local structural distortions.

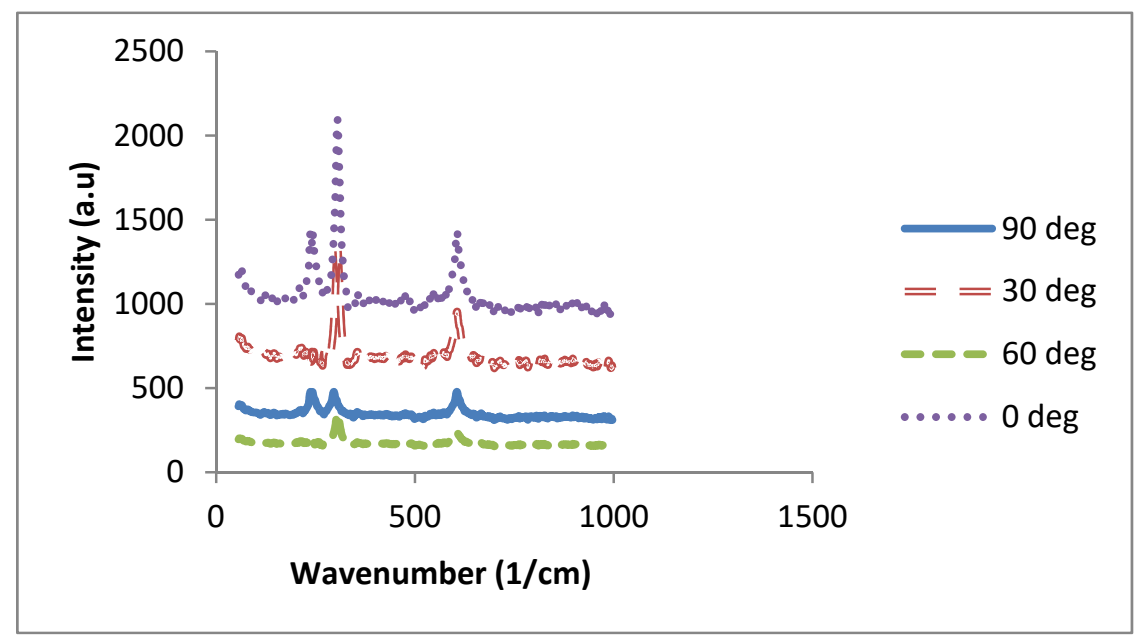

Figure 8. Experimental data for Raman spectra with different angles. $\boldsymbol{\theta}$ denotes the angle between the long axis of the nanowire ( $\mathrm{z}$ axis) and the laser polarization

\section{Conclusion}

There are many ways to grow CdS nanowires but growing vertically aligned nanowires has always been a challenge. This paper discusses about the most economical way is to grow vertically aligned nanowires in a nanoporous template through dc electrodeposition by overcoming the bottlenecks of barrier layer removal during the growth of CdS nanowires which can sometimes lead to poor crystallinity of grown CdS nanowires. CdS nanowires were self assembled in an anodized aluminum sheet metal of thickness of $500 \mu \mathrm{m}$. The polarization properties of the $\mathrm{CdS}$ nanowires were investigated for their use in polarization sensitive optoelectronic devices. Also, the PL peaks were in accordance to the bandgap of CdS nanowires showing good crystalline growth of $\mathrm{CdS}$ nanowires via dc electrodeposition. Polarization-sensitive measurements reveal a striking anisotropy in the 
PL intensity recorded parallel and perpendicular to the long axis of a nanowire. The measured PL ratio, $\rho$, is around 0.80-0.85 indicating very good polarization anisotropy for CdS nanowires. The highest polarization ratio observed previously for CdS nanowires is only 0.78 (Acharya, Patla, Kost, Efrima, \& Golan, 2006; Robert et al., 2014) (H. M. Fan, Ni, Feng, Fan, Shen, et al., 2007). These nanowire arrays can be used as optical switches as a result of their polarization anisotropy properties. This research may pave the way for an economical fabrication of polarization sensitive nanoscale devices with high performance. The observed peaks were slightly different for CdS NWs than the reported frequencies of CdS bulk crystals given in Table 2. The 1 LO phonon energy has been observed more for CdS NWs as compared to CdS bulk. The same behavior with the increase in 1 LO phonon energy was also reported in Refs. (Fu, Li, \& Tang; Pan et al.). Also, good crystallinity enhances multiphonon responses due to well-defined phonons in the material. The optical properties of CdS nanowires are also influenced greatly by orientation and polarization angle as seen in the paper. Hence, CdS nanowires with good crystallinity can be grown using simple methods such as dc electrodeposition making it possible to use them for its use in high performance nanowire polarizer application.

\section{Acknowledgments}

We gratefully acknowledge that anodized alumina templates were grown in Brown University by one of us (S.D.) under the supervision of Prof. Jimmy $\mathrm{Xu}$ and this work was partially supported by AFOSR FA9550-11-1-0271.

\section{References}

Acharya, S., Patla, I., Kost, J., Efrima, S., \& Golan, Y. (2006). Switchable Assembly of Ultra Narrow CdS Nanowires and Nanorods. Journal of the American Chemical Society, 128(29), 9294-9295. https://doi.org/10. 1021/ja062404i

Agarwal, R., Barrelet, C. J., \& Lieber, C. M. (2005). Lasing in single cadmium sulfide nanowire optical cavities. Nano Letters, 5(5), 917-920. https://doi.org/10.1021/N1050440u

Anyebe, E. A., Sanchez, A. M., Hindmarsh, S., Chen, X., Shao, J., Rajpalke, M. K., . . Z Zhuang, Q. (2015). Realization of Vertically Aligned, Ultrahigh Aspect Ratio InAsSb Nanowires on Graphite. Nano Letters, 15(7), 4348-4355. https://doi.org/10.1021/acs.nanolett.5b00411

Arguello, C. A., Rousseau, D. L., \& Porto, S. P. S. (1969). First-Order Raman Effect in Wurtzite-Type Crystals. Physical Review, 181(3), 1351-\&. https://doi.org/10.1103/Physrev.181.1351

Brenneman, K. L., Poduri, S., Stroscio, M. A., \& Dutta, M. (2013). Optical detection of lead (II) ions using DNA-based nanosensor. IEEE Sensors Journal, 13(5), 1783-1786.

Duan, X. F., Huang, Y., Agarwal, R., \& Lieber, C. M. (2003). Single-nanowire electrically driven lasers. Nature, 421(6920), 241-245. https://doi.org/10.1038/Nature01353

Dutta, M., Stroscio, M. A., Qian, J., Wu, T.-C., Sen, B., Zhang, N., . . Shukla, P. (2014). Semiconductor Quantum Dots: Nanosensors Based on DNA and RNA Aptamers Dekker Encyclopedia of Nanoscience and Nanotechnology, Third Edition (pp. 4383-4389). CRC Press.

Fan, H. M., Fan, X. F., Ni, Z. H., Shen, Z. X., Feng, Y. P., \& Zou, B. S. (2008). Orientation-dependent Raman spectroscopy of single wurtzite CdS nanowires. Journal of Physical Chemistry C, 112(6), 1865-1870. https://doi.org/10.1021/Jp7096839

Fan, H. M., Ni, Z. H., Feng, Y. P., Fan, X. F., Kuo, J. L., Shen, Z. X., \& Zou, B. S. (2007). Anisotropy of electron-phonon coupling in single wurtzite CdS nanowires. Applied Physics Letters, 91(17). https://doi.org/10. 1063/1.2805380

Fan, H. M., Ni, Z. H., Feng, Y. P., Fan, X. F., Shen, Z. X., \& Zou, B. S. (2007). High-pressure Raman and photoluminescence of highly anisotropic CdS nanowires. Journal of Raman Spectroscopy, 38(9), 1112-1116. https://doi.org/10.1002/jrs.1724

Fan, Z. Y., Chang, P. C., Lu, J. G., Walter, E. C., Penner, R. M., Lin, C. H., \& Lee, H. P. (2004). Photoluminescence and polarized photodetection of single $\mathrm{ZnO}$ nanowires. Applied Physics Letters, 85(25), 6128-6130. https://doi.org/10.1063/1.1841453

Farid, S., Meshik, X., Choi, M., Mukherjee, S., Lan, Y., Parikh, D., . . W Wang, Y. Y. (2015). Detection of Interferon gamma using graphene and aptamer based FET-like electrochemical biosensor. Biosensors and Bioelectronics, 71, 294-299. 
Fu, X. L., Li, L. H., \& Tang, W. H. (2006). Preparation and characterization of CdS/Si coaxial nanowires. Solid State Communications, 138(3), 139-142. https://doi.org/10.1016/J.Ssc.2006.02.015

Gu, Y., Kwak, E. S., Lensch, J. L., Allen, J. E., Odom, T. W., \& Lauhon, L. J. (2005). Near-field scanning photocurrent microscopy of a nanowire photodetector. Applied Physics Letters, 87(4). https://doi.org/10. $1063 / 1.1996851$

Gudiksen, M. S., Wang, J., \& Lieber, C. M. (2002). Size-Dependent Photoluminescence from Single Indium Phosphide Nanowires. The Journal of Physical Chemistry B, 106(16), 4036-4039. https://doi.org/10.1021/ jp014392n

Ils, P., Greus, C., Forchel, A., Kulakovskii, V. D., Gippius, N. A., \& Tikhodeev, S. G. (1995). Linear-Polarization of Photoluminescence Emission and Absorption in Quantum-Well Wire Structures Experiment and Theory. Physical Review B, 51(7), 4272-4277. https://doi.org/10.1103/Physrevb.51.4272

Kind, H., Yan, H., Messer, B., Law, M., \& Yang, P. (2002). Nanowire Ultraviolet Photodetectors and Optical Switches. Advanced Materials, 14(2), 158-160. https://doi.org/10.1002/1521-4095(20020116)14:2<158:: AID-ADMA158>3.0.CO;2-W

Lee, B. C., Kim, K. W., Dutta, M., \& Stroscio, M. A. (1997). Electron-optical-phonon scattering in wurtzite crystals. Physical Review B, 56(3), 997-1000. https://doi.org/10.1103/Physrevb.56.997

Mazouchi, M., Poduri, S., \& Dutta, M. (2014). Growth and Characterization of Indium Oxide, Zinc Oxide and Cadmium Sulfide Nanowires by Vapor-Liquid-Solid Growth Technique. Applied Physics Research, 6(6), 55 .

Morales, A. M., \& Lieber, C. M. (1998). A laser ablation method for the synthesis of crystalline semiconductor nanowires. Science, 279(5348), 208-211. https://doi.org/10.1126/Science.279.5348.208

Mukherjee, S., Meshik, X., Choi, M., Farid, S., Datta, D., Lan, Y., ... \& Wang, Y. Y. (2015). A graphene and aptamer based liquid gated FET-like electrochemical biosensor to detect adenosine triphosphate. IEEE transactions on nanobioscience, 14(8), 967-972.

Pan, A. L., Liu, R. B., Yang, Q., Zhu, Y. C., Yang, G. Z., Zou, B. S., \& Chen, K. Q. (2005). Stimulated emissions in aligned CdS nanowires at room temperature. Journal of Physical Chemistry B, 109(51), 24268-24272. https://doi.org/10.1021/Jp055164m

Poduri, S. D. (2010). Theoretical Modeling and Analysis of Ammonia Gas Sensing Properties of Vertically Aligned Multiwalled Carbon Nanotube Resistive Sensors and Enhancing Their Sensitivity.

Poduri, S., Dutta, M., \& Stroscio, M. (2014). Characterization of CdS Nanowires Self-Assembled in a Nanoporous Alumina Template. Journal of Electronic Materials, 43(11), 3979-3983. https://doi.org/10.1007/S11664-014-3305-0

Qian, F., Gradecak, S., Li, Y., Wen, C. Y., \& Lieber, C. M. (2005). Core/multishell nanowire heterostructures as multicolor, high-efficiency light-emitting diodes. Nano Letters, 5(11), 2287-2291. https://doi.org/10.1021/ N1051689e

Robert, R., Daniel, P., Arian, K., Robert, B., Sebastian, G., Ulf, P., \& Carsten, R. (2014). Polarization features of optically pumped CdS nanowire lasers. Journal of Physics D: Applied Physics, 47(39), 394012.

Routkevitch, D., Bigioni, T., Moskovits, M., \& Xu, J. M. (1996). Electrochemical Fabrication of CdS Nanowire Arrays in Porous Anodic Aluminum Oxide Templates. The Journal of Physical Chemistry, 100(33), 14037-14047. https://doi.org/10.1021/jp952910m

Routkevitch, D., Bigioni, T., Moskovits, M., \& Xu, J. M. (1996). Electrochemical fabrication of CdS nanowire arrays in porous anodic aluminum oxide templates. Journal of Physical Chemistry, 100(33), 14037-14047. https://doi.org/10.1021/Jp952910m

Ruda, H. E., \& Shik, A. (2005). Scanning capacitance microscopy of nanostructures - art. no. 075315. Physical Review B, 71(7). https://doi.org/10.1103/Physrevb.71.075316

S. Poduri, M. S. C., Mitra Dutta, \& Stroscio, M. (2015). Numerical analysis of electric field enhancement in ZnO film with plasmonic au quantum dots. Paper presented at the IEEE International Workshop Conference of Computational Electronics. 
Samuelson, L., Bjork, M. T., Deppert, K., Larsson, M., Ohlsson, B. J., Panev, N., . . Wallenberg, L. R. (2004). Semiconductor nanowires for novel one-dimensional devices. Physica E-Low-Dimensional Systems \& Nanostructures, 21(2-4), 560-567. https://doi.org/10.1016/J.Physe.2003.11.072

Schmidt, T., Lischka, K., \& Zulehner, W. (1992). Excitation-power dependence of the near-band-edge photoluminescence of semiconductors. Physical Review B, 45(16), 8989-8994.

Seminario, J. M. (2014). Design and Applications of Nanomaterials for Sensors (Vol. 16): Springer.

Shan, C. X., Liu, Z., \& Hark, S. K. (2006). Photoluminescence polarization in individual CdSe nanowires. Physical Review B, 74(15). https://doi.org/10.1103/Physrevb.74.153402

Taşaltın, N., Öztürk, S., Kılınç, N., Yüzer, H., \& Öztürk, Z. (2010). Fabrication of vertically aligned Pd nanowire array in AAO template by electrodeposition using neutral electrolyte. Nanoscale Research Letters, 5(7), 1137-1143. https://doi.org/10.1007/s11671-010-9616-Z

Vouilloz, F., Oberli, D. Y., Dupertuis, M. A., Gustafsson, A., Reinhardt, F., \& Kapon, E. (1998). Effect of lateral confinement on valence-band mixing and polarization anisotropy in quantum wires. Physical Review B, 57(19), 12378-12387. https://doi.org/10.1103/Physrevb.57.12378

Wang, J. F., Gudiksen, M. S., Duan, X. F., Cui, Y., \& Lieber, C. M. (2001). Highly polarized photoluminescence and photodetection from single indium phosphide nanowires. Science, 293(5534), 1455-1457. https://doi.org/ 10.1126/Science.1062340

Xiang, J., Lu, W., Hu, Y., Wu, Y., Yan, H., \& Lieber, C. M. (2006). Ge/Si nanowire heterostructures as high-performance field-effect transistors. Nature, 441(7092), 489-493. https://doi.org/10.1038/nature04796

Xu, D. S., Xu, Y. J., Chen, D. P., Guo, G. L., Gui, L. L., \& Tang, Y. Q. (2000). Preparation and characterization of CdS nanowire arrays by dc electrodeposit in porous anodic aluminum oxide templates. Chemical Physics Letters, 325(4), 340-344. https://doi.org/10.1016/S0009-2614(00)00676-X

Xu, K., Purahmad, M., Brenneman, K., Meshik, X., Farid, S., Poduri, S., . . . Dutta, M. (2014). Design and Applications of Nanomaterial-Based and Biomolecule-Based Nanodevices and Nanosensors. In J. M. Seminario (Ed.), Design and Applications of Nanomaterials for Sensors (pp. 61-97). Dordrecht: Springer Netherlands.

Yang, Y., Chen, H. L., Mei, Y. F., Chen, J. B., Wu, X. L., \& Bao, X. M. (2002). Anodic alumina template on $\mathrm{Au} / \mathrm{Si}$ substrate and preparation of CdS nanowires. Solid State Communications, 123(6-7), 279-282. https://doi.org/S0038-1098(02)00304-6 Doi 10.1016/S0038-1098(02)00304-6

Zhou, W., Zhang, J., Li, X., Liu, Y., Min, G., Song, Z., \& Zhang, J. (2009). Replication of mold for UV-nanoimprint lithography using AAO membrane. Applied Surface Science, 255(18), 8019-8022. https://doi.org/10.1016/j.apsusc.2009.05.006

\section{Copyrights}

Copyright for this article is retained by the author(s), with first publication rights granted to the journal.

This is an open-access article distributed under the terms and conditions of the Creative Commons Attribution license (http://creativecommons.org/licenses/by/4.0/). 